\title{
Comparison of Mid-Level Feature Coding Approaches And Pooling Strategies in Visual Concept Detection (Supplementary Material).
}

\author{
P. Koniusz, F. Yan, K. Mikolajczyk \\ Centre for Vision, Speech and Signal Processing, University of Surrey, GU2 7XH, Guildford, UK
}

\begin{abstract}
A number of techniques for generating mid-level features, including two variants of Soft Assignment, Locality-constrained Linear Coding, and Sparse Coding, are evaluated in the main document [1]. Pooling methods that aggregate mid-level features into vectors representing images like Average pooling, Max-pooling, and a family of likelihood inspired pooling strategies are scrutinised there. This supplementary material extends our evaluations to the PascalVOC07 dataset given Sparse Coding, as state-of-the-art classification performance it the main document is demonstrated thus far on Caltech101, Flower17, and ImageCLEF11 datasets.
\end{abstract}

Keywords: Bag-Of-Words, Mid-level features, Soft Assignment, Sparse Coding, Locality-constrained Linear Coding, Max-pooling, Analytical Pooling, Power Normalisation, Comparison

\section{Experimental Arrangements}

Sparse Coding $[2,3]$ (SC) is evaluated on the PascalVOC07 [4] dataset. Online Dictionary Learning is used to train dictionaries for this experiment [5]. The spatial relations in images are exploited by either Spatial Coordinate Coding [6, 1] (SCC) or Spatial Pyramid Matching [7] (SPM). Dominant Angle Pyramid Matching [6, 1] (DoPM) that exploits orientations of dominant edges from the local descriptors is also evaluated. SPM is set to 3 levels of coarseness with $1 \times 1,1 \times 3,3 \times 1$, and $2 \times 2$ grids. DoPM is set to 5 levels of coarseness with $1,3,6,9$, and 12 grids. Moreover, DoPM employs SCC by default.

The mid-level features are aggregated by Max-pooling [3] (Max), Power Normalisation [8] (Gamma), theoretical expec-

Email address: p.koniusz@surrey .ac.uk (P. Koniusz)

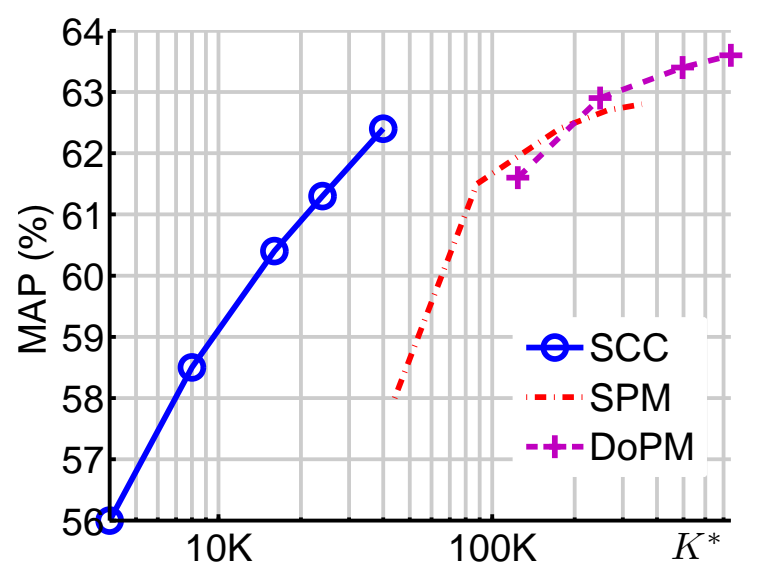

Figure 1: Evaluation of SCC, SPM, and DoPM approaches on the PascalVOC07 set. The overall signature length $K^{*}$ is indicated. Linear kernels and MaxExp@n=7 are used for this experiment. tation of Max-pooling [9] (MaxExp), its linearised approximation [1] (AxMin), and the @n scheme [1] combined with MaxExp (MaxExp@n). Note that the@n scheme combined with AxMin (AxMin@n) is evaluated in the main document [1]. Moreover, linear kernels are used in the following experiments. Multi-label KDA [10] is applied on PascalVOC07, as it was previously found to be a robust performer on this set. Mean Average Precision [10] (MAP) is used to report the classification performance. Table 1 details the experimental parameters.

\section{Evaluations on PascalVOC07}

Figure 1 compares the classification performance of SCC, SPM, and DoPM approaches on the PascalVOC07 set given various dictionary sizes. Linear kernels and MaxExp@ $n=7$ are used for this experiment. The dictionary size is varied from 4000 to 40000 atoms for SCC. The signature lengths $K^{*}$ are the same as the dictionary sizes. The highest result attained by SCC amounts to $62.4 \%$ MAP. Moreover, we vary the dictionary size from 4000 to 32000 atoms for SPM. This results in the signature lengths between $K^{*}=44000$ and $K^{*}=352000$. The best

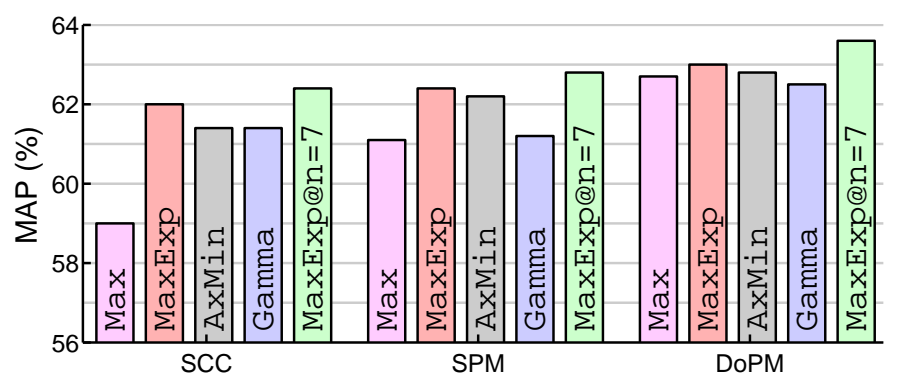

Figure 2: Evaluation of SCC, SPM, and DoPM schemes on the PascalVOC07 set given Max-pooling, MaxExp, AxMin, Gamma, and MaxExp@n=7. The dictionary sizes are 40000, 32000, and 24000 atoms for SCC, SPM, and DoPM. 


\begin{tabular}{c|c|c|c|c|c|c|c|}
\multirow{3}{*}{ Dataset } & $\begin{array}{c}\text { Splits } \\
\text { no. }\end{array}$ & \multicolumn{2}{|c|}{$\begin{array}{c}\text { Train+Val. } \\
\text { samples }\end{array}$} & $\begin{array}{c}\text { Test } \\
\text { samples }\end{array}$ & $\begin{array}{c}\text { Total } \\
\text { images }\end{array}$ & $\begin{array}{c}\text { Dict. } \\
\text { size }\end{array}$ & $\begin{array}{c}\text { Descr. type/ } \\
\text { dimensions }\end{array}$ \\
\hline \multirow{3}{*}{ PascalVOC07 } & 1x & $2501+2510=5011$ & 4952 & 9963 & 4K-40K & SIFT/128D \\
\cline { 2 - 7 } & $\begin{array}{c}\text { Descr. } \\
\text { interval }(\mathrm{px})\end{array}$ & $\begin{array}{c}\text { Radii } \\
(\mathrm{px})\end{array}$ & $\begin{array}{c}\text { Descr. } \\
\text { per img. }\end{array}$ & $\begin{array}{c}\text { Spatial/other } \\
\text { schemes }\end{array}$ & $\begin{array}{c}\text { Kernel } \\
\text { types }\end{array}$ & $\begin{array}{c}\text { Classifier } \\
\text { used }\end{array}$ \\
\cline { 2 - 7 } & $4,6,8,10,12,14,16$ & $12,16,24,32,40,48,56$ & 19420 & SCC/SPM/DOPM & linear & multilabel \\
\hline
\end{tabular}

Table 1: Summary of the descriptor parameters and various experimental details.

result attained by SPM amounts to $62.8 \%$ MAP. Lastly, the dictionary size is varied from 4000 to 24000 atoms for DoPM. The corresponding signature lengths are between $K^{*}=124000$ and $K^{*}=744000$. This method scores $63.6 \%$ MAP.

Figure 2 demonstrates various pooling strategies given dictionary sizes of 40000, 32000, and 24000 atoms for SCC, SPM, and DoPM approaches, respectively. Firstly, we discuss SCC approach. MaxExp@n=7 scores 62.4\% MAP followed closely by MaxExp that yields $62.0 \%$ MAP. AxMin and Gamma attain the same score of $61.4 \%$ MAP followed by Max-pooling that yields 59.0\% MAP only.

Next, we discuss SPM approach. MaxExp@ $n=7$ scores 62.8\% MAP followed closely by MaxExp and AxMin that yield $62.4 \%$ and $62.2 \%$ MAP. Gamma and Max-pooling attain $61.2 \%$ and $61.1 \%$ MAP only.

Lastly, we discuss DoPM approach. MaxExp@ $n=7$ scores 63.6\% MAP followed by MaxExp and AxMin that yield 63.0\% and $62.8 \%$ MAP. Max-pooling attains $62.7 \%$ MAP and outperforms Gamma that yields $62.5 \%$ MAP only.

\section{Conclusions}

SCC approach results in very competitive signature lengths. However, the coding step is computationally prohibitive for large visual dictionaries. It takes 815 and 3.6 seconds to code 1000 descriptors on a single $2.3 \mathrm{GHz}$ AMD Opteron core given $K=$ 40000 and $K=4000$ atoms, respectively. This may be partially addressed by Fast Hierarchical Nearest Neighbour Search (FHNNS) proposed in the main document [1]. SPM achieves a marginally better performance with somewhat smaller dictionaries at a price of larger image signatures. DoPM achieves the best performance at a price of sizeable image signatures.

Furthermore, we observe that the @ $n$ scheme (combined with MaxExp) attains the highest scores amongst the investigated pooling strategies. MaxExp and its approximation AxMin are also strong performers followed by Gamma and Max-pooling. These results are consistent with the main observations in [1].

\section{References}

[1] P. Koniusz, F. Yan, K. Mikolajczyk, Comparison of Mid-Level Feature Coding Approaches And Pooling Strategies in Visual Concept Detection, CVIU (2012).

[2] H. Lee, A. Battle, R. Raina, A. Y. Ng, Efficient Sparse Coding Algorithms, NIPS (2007) 801-808.

[3] J. Yang, K. Yu, Y. Gong, T. S. Huang, Linear Spatial Pyramid Matching using Sparse Coding for Image Classification, CVPR (2009) 1794-1801.
[4] M. Everingham, L. Van Gool, C. K. I. Williams, J. Winn, A. Zisserman, The PASCAL Visual Object Classes Challenge 2007 (VOC2007) Results, http://pascallin.ecs.soton.ac.uk/challenges/VOC, 2007.

[5] J. Mairal, F. Bach, J. Ponce, G. Sapiro, Online Learning for Matrix Factorization and Sparse Coding, JMLR (2010).

[6] P. Koniusz, K. Mikolajczyk, Spatial Coordinate Coding to Reduce Histogram Representations, Dominant Angle and Colour Pyramid Match, ICIP (2011).

[7] S. Lazebnik, C. Schmid, J. Ponce, Beyond Bags of Features: Spatial Pyramid Matching for Recognizing Natural Scene Categories, CVPR 2 (2006) 2169-2178.

[8] F. Perronnin, J. Sánchez, T. Mensink, Improving the Fisher Kernel for Large-Scale Image Classification, ECCV (2010) 143-156.

[9] Y. Boureau, J. Ponce, Y. LeCun, A Theoretical Analysis of Feature Pooling in Vision Algorithms, ICML (2010).

[10] M. Tahir, J. Kittler, K. Mikolajczyk, F. Yan, K. van de Sande, T. Gevers, Visual Category Recognition using Spectral Regression and Kernel Discriminant Analysis, ICCV Workshop on Subspace Methods (2009). 